\title{
Information Dissemination of Two Ways on Complex Networks
}

\author{
Chen Wenbin ${ }^{1, a}$, Qin Peng ${ }^{2, b}$ \\ ${ }^{1}$ Shanxi Provincial Key Laboratory for Biomedical Imaging and Big Data, North University of \\ China,Taiyuan, China \\ ${ }^{2}$ School of Electrical and Control Engineering, North University of China,Taiyuan, China \\ azbdxcwb@163.com, bqinpeng@nuc.edu.cn
}

Keywords: information dissemination; complex network; reproduction number; stability

\begin{abstract}
An SIS information dissemination model is studied based in social network. QQ and WeChat are employed as the carrier of information dissemination. We assume that uninformed individuals can get information from either their informed QQ friends or their informed WeChat friends, and the relationship between two individuals can only be QQ relationship or WeChat relationship. Informed individuals forget the information at a certain probability. Basic reproduction number was obtained by mathematical analysis. Global stability of the disease-free equilibrium and the existence and uniqueness of endemic equilibrium were demonstrated. To illustrate our theoretical analysis, some numerical simulations are included in this paper.
\end{abstract}

\section{Introduction}

In recent years, studies of information dissemination in complex networks have attracted tremendous interest among the public. These studies assume that the population can be represented by complex networks. Nodes represent individuals, while edges represent all kinds of interactions among individuals, and information spreads along the edges of social network.

In fact, many social, biological, and communication systems can be described as complex networks. As representatives of online social-networking, two kinds of online chat tools, QQ and WeChat are employed as the carrier of information dissemination in China.

To date, most researches have focused on negative information, such as rumors. For example, Sudbury analyzed the spread of rumor ${ }^{[2]}$ with epidemic SIR model, three types of individuals were divided into during rumor dissemination, and he gave the definition of each type. Sudbury also researched the model on the random network, and discovered that the number of rumors has a ceiling $^{[2]}$. Zanette ${ }^{[3,4]}$ and Moreno ${ }^{[1]}$ respectively studied the rumor dissemination model by means of the mean field method on the small world network ${ }^{[3,4]}$ and scale-free networks ${ }^{[1]}$, and they discovered that people bewitched by rumors are fewer comparing with the random network under the same circumstances. Zonghua Liu et al further proved that the random network is easy to spread rumors among common networks ${ }^{[5,6]}$. Recently, Kesten et al studied the spreading process of rumors in the mobile population using the rigorous probability theory, where the human population was regarded as the multi particle system of Brownian motion ${ }^{[7]}$.

In this paper, an information dissemination SIS model based on BA scale-free network is built and the global asymptotical stability of the disease-free equilibrium is discussed.

\section{Model and parameter}

We suppose that information disseminates by communicating with either QQ friends or WeChat friends. All individuals are classified into two discrete states: S-state(the uninformed individuals); Istate(the active informed individuals, who are possible to transmit information to their $\mathrm{S}$ neighbors). Information spreading process is described below. Step 1, S individuals get information with 
probability $\lambda_{1}$ if they communicate with their informed QQ friends, and also get information with probability $\lambda_{2}$ if they communicate with their informed WeChat friends. Step 2, I individuals forget information and become $\mathrm{S}$ individuals with a certain probability $\gamma$.Taking into account the heterogeneity induced by the nodes with different degree $\left(k_{1}, k_{2}\right)$, we divide the nodes into different classes, and the nodes in each class have the same degree $\left(k_{1}, k_{2}\right) \cdot S_{\left(k_{1}, k_{2}\right)}(t)$ represents the number of $S$ individuals that have $k_{1}$ QQ friends and $k_{2}$ WeChat friends at time $t \cdot I_{\left(k_{1}, k_{2}\right)}(t)$ represents the number of $I$ individuals that have $k_{1}$ QQ friends and $k_{2}$ WeChat friends at time $t$. Total population $N_{\left(k_{1}, k_{2}\right)}(t)=S_{\left(k_{1}, k_{2}\right)}(t)+I_{\left(k_{1}, k_{2}\right)}(t)$ is a constant.

The flow chart of the model is shown in Figure 1, and the frame diagram is shown in Figure 2.

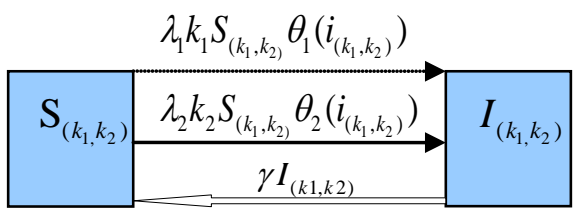

Figure 1. State-transition rules of the contact process.

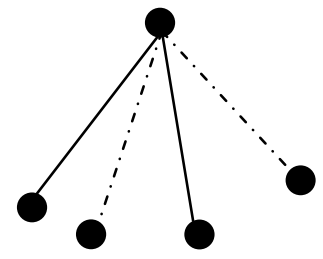

Figure 2. QQ friends are connected by dotted line, WeChat friends are connected by solid line.

TABLE I. SYMBOL TABLE

\begin{tabular}{|c|c|}
\hline Parameter & Definition \\
\hline$S_{\left(k_{1}, k_{2}\right)}(t)$ & $\begin{array}{l}\text { the number of uninformed individuals that have } k_{1} \\
\text { QQ friends and } k_{2} \text { WeChat friends at time } t\end{array}$ \\
\hline$I_{\left(k_{1}, k_{2}\right)}(t)$ & $\begin{array}{l}\text { the number of informed individuals that have } k_{1} \text { QQ } \\
\text { friends and } k_{2} \text { WeChat friends at time } t\end{array}$ \\
\hline$N_{\left(k_{1}, k_{2}\right)}(t)$ & $\begin{array}{l}\text { the number of individuals that have } k_{1} \text { QQ friends } \\
\text { and } k_{2} \text { WeChat friends at time } t\end{array}$ \\
\hline$\lambda_{1}$ & $\begin{array}{l}\text { the probability of uninformed individuals to get } \\
\text { information from their informed QQ friends }\end{array}$ \\
\hline$\lambda_{2}$ & $\begin{array}{l}\text { the probability of uninformed individuals to get } \\
\text { information from their informed WeChat friends }\end{array}$ \\
\hline$\gamma$ & $\begin{array}{c}\text { the probability of informed individuals to forget } \\
\text { information }\end{array}$ \\
\hline
\end{tabular}

Based on above assumption, the dynamic equation of SIS information dissemination model of two kinds of dissemination modes is written as follow:

$$
\left\{\begin{aligned}
\frac{d S_{\left(k_{1}, k_{2}\right)}(t)}{d t}= & -\lambda_{1} k_{1} S_{\left(k_{1}, k_{2}\right)}(t) \theta_{1}\left(i_{\left(k_{1}, k_{2}\right)}\right)(t) \\
& -\lambda_{2} k_{2} S_{\left(k_{1}, k_{2}\right)}(t) \theta_{2}\left(i_{\left(k_{1}, k_{2}\right)}\right)(t)+\gamma I_{\left(k_{1}, k_{2}\right)}(t) \\
\frac{d I_{\left(k_{1}, k_{2}\right)}(t)}{d t}= & \lambda_{1} k_{1} S_{\left(k_{1}, k_{2}\right)}(t) \theta_{1}\left(i_{\left(k_{1}, k_{2}\right)}\right)(t) \\
& +\lambda_{2} k_{2} S_{\left(k_{1}, k_{2}\right)}(t) \theta_{2}\left(i_{\left(k_{1}, k_{2}\right)}\right)(t)-\gamma I_{\left(k_{1}, k_{2}\right)}(t)
\end{aligned}\right.
$$


The parameters in the above equation are described in Table 1.

$$
\begin{gathered}
\text { Where, } \theta_{1}(\mathrm{t})=\frac{\sum_{k_{1}=0}^{n} \sum_{k_{2}=0}^{n} k_{1} I_{\left(k_{1}, k_{2}\right)}(t)}{\sum_{k_{1}=0}^{n} \sum_{k_{2}=0}^{n}\left(k_{1}+k_{2}\right) N_{\left(k_{1}, k_{2}\right)}(t)}, \theta_{1}(\mathrm{t})=\frac{\sum_{k_{1}=0}^{n} \sum_{k_{2}=0}^{n} k_{1} I_{\left(k_{1}, k_{2}\right)}(t)}{\sum_{k_{1}=0}^{n} \sum_{k_{2}=0}^{n}\left(k_{1}+k_{2}\right) N_{\left(k_{1}, k_{2}\right)}(t)}, \\
P\left(k_{1}, k_{2}\right) \text { is the probability that a random node has the degree }\left(k_{1}, k_{2}\right) \text {. } \\
S_{\left(k_{1}, k_{2}\right)}(t)=\frac{S_{\left(k_{1}, k_{2}\right)}(t)}{N_{\left(k_{1}, k_{2}\right)}(t)}, i_{\left(k_{1}, k_{2}\right)}(t)=\frac{I_{\left(k_{1}, k_{2}\right)}(t)}{N_{\left(k_{1}, k_{2}\right)}(t)} \text {. }
\end{gathered}
$$

The marginal distributions:

$$
\sum_{\mathrm{k}_{1}=0}^{n} \sum_{k_{2}=0}^{n} k_{1} P\left(k_{1}, k_{2}\right)(t)=<k_{1}>, \sum_{\mathrm{k}_{1}=0}^{n} \sum_{k_{2}=0}^{n} k_{2} P\left(k_{1}, k_{2}\right)(t)=<k_{2}>
$$

So, the system (1) can be written as the following equations:

$$
\begin{aligned}
\frac{\mathrm{di}_{\left(k_{1}, k_{2}\right)}(t)}{d t}= & \lambda_{1} k_{1}\left(1-\mathrm{i}_{\left(k_{1}, k_{2}\right)}(t)\right) \times \\
& \frac{\sum_{k_{1}=0}^{n} \sum_{k_{2}=0}^{n} k_{1} P\left(k_{1}, k_{2}\right)(t) \mathrm{i}_{\left(k_{1}, k_{2}\right)}(t)}{<k_{1}+\mathrm{k}_{2}>} \\
& +\lambda_{2} k_{2}\left(1-\mathrm{i}_{\left(k_{1}, k_{2}\right)}(t)\right) \times \\
& \frac{\sum_{k_{1}=0}^{n} \sum_{k_{2}=0}^{n} k_{2} P\left(k_{1}, k_{2}\right)(t) \mathrm{i}_{\left(k_{1}, k_{2}\right)}(t)}{<k_{1}+\mathrm{k}_{2}>} \\
& -\gamma \mathrm{i}_{\left(k_{1}, k_{2}\right)}(t)
\end{aligned}
$$

Without loss of generality, we set $\gamma=1$.

\section{The analysis of model}

\subsection{Basic reproduction number}

An important epidemiological parameter is $R_{0}$, the basic reproduction number. According to ${ }^{[8,9]}$, which can be computed by $R_{0}=\rho\left(F V^{-1}\right)$, where $\rho(M)$ represents the spectral radius of matrix $M$, $F$ is the rate of appearance of new infections, and $V$ is the rate of transfer of individuals out of the compartments. Then

$$
F=F_{\mathrm{ij}}^{(n+1) \times(n+1)} \cdot i, j=1,2, \ldots,(n+1) .
$$

In which, $V=V_{i j}^{(n+1) \times(n+1)}$ is an identity matrix. The definition of $F_{i j}$ is shown in (3).

Apply similar transformation to matrix $\boldsymbol{F}$ :

First, apply line transformation to $\boldsymbol{F}$,

(1) $\mathrm{r}_{3}+\mathrm{r}_{2} \times(-1), \cdots, \mathrm{r}_{(n+1)}+\mathrm{r}_{2} \times(-1), \mathrm{r}_{\mathrm{i}(n+1)+3}+\mathrm{r}_{\mathrm{i}(\mathrm{n}+1)+2} \times(-2)$,

(1) $\cdots, \mathrm{r}_{(i+1)(n+1)}+\mathrm{r}_{\mathrm{i}(n+1)} \times(-n)$ for $i=1,2, \cdots, n$.

(2) $r_{i(n+1)+2}+r_{2} \times(-1)$ for $i=1,2, \cdots, n$.

(3) $r_{i(n+1)+1}+r_{(n+1)+1} \times(-i)$ for $i=1,2, \cdots, n$.

(4) $\mathrm{r}_{j(n+1)+2}+r_{2} \times(-1)$ for $i=1,2, \cdots, n$.

(5) exchanging of $r_{1}$ with $r_{(n+1)+1}$. 


$$
\boldsymbol{F}_{i j}=\left(\begin{array}{cccc}
\frac{(i-1)(j-1) \mathrm{p}(j-1,0) \lambda_{1}}{<k_{1}>+<k_{2}>} & \frac{(i-1)(j-1) \mathrm{p}(j-1,1) \lambda_{1}}{<k_{1}>+<k_{2}>} & \cdots & \frac{(i-1)(j-1) \mathrm{p}(j-1, n) \lambda_{1}}{<k_{1}>+<k_{2}>} \\
\frac{(i-1)(j-1) \mathrm{p}(j-1,0) \lambda_{1}}{<k_{1}>+<k_{2}>} & \frac{(i-1)(j-1) \mathrm{p}(j-1,1) \lambda_{1}+\mathrm{p}(j-1,1) \lambda_{2}}{<k_{1}>+<k_{2}>} & \cdots & \frac{(i-1)(j-1) \mathrm{p}(j-1, n) \lambda_{1}+n \mathrm{p}(j-1, n) \lambda_{2}}{<k_{1}>+<k_{2}>} \\
\vdots & \vdots & \vdots & \vdots \\
\frac{(i-1)(j-1) \mathrm{p}(j-1,0) \lambda_{1}}{<k_{1}>+<k_{2}>} & \frac{(i-1)(j-1) \mathrm{p}(j-1,1) \lambda_{1}+n \mathrm{p}(j-1, n) \lambda_{2}}{<k_{1}>+<k_{2}>} & \cdots & \frac{(i-1)(j-1) \mathrm{p}(j-1, n) \lambda_{1}+n^{2} \mathrm{p}(j-1, n) \lambda_{2}}{<k_{1}>+<k_{2}>}
\end{array}\right)
$$

Then, apply rank transformation, the matrix $\boldsymbol{F}$ is transformed into

$$
\overline{\boldsymbol{F}}=\left(\begin{array}{cc}
F^{*} & F^{* *} \\
0 & 0
\end{array}\right)
$$

Where

$$
\boldsymbol{F}^{*}=\left(\begin{array}{ll}
\frac{\sum_{i=1}^{n} \sum_{j=0}^{n} i^{2} p(i, j) \lambda_{1}}{<k_{1}>+<k_{2}>} & \frac{\sum_{i=1}^{n} \sum_{j=1}^{n} i j p(i, j) \lambda_{1}}{<k_{1}>+<k_{2}>} \\
\frac{\sum_{i=1}^{n} \sum_{j=1}^{n} i j p(i, j) \lambda_{2}}{<k_{1}>+<k_{2}>} & \frac{\sum_{i=0}^{n} \sum_{j=1}^{n} j^{2} p(i, j) \lambda_{2}}{<k_{1}>+<k_{2}>}
\end{array}\right)
$$

The discriminant of matrix $\boldsymbol{F}^{*}$ is greater than 0 , so the matrix $\boldsymbol{F}^{*}$ has two different real characteristic roots.

By utilizing the method proposed, ${ }^{[9]}$ we know the basic reproduction number $R_{0}=\rho\left(F V^{-1}\right)$ is the maximum value of the characteristic roots of the matrix $\overline{\boldsymbol{F}}$, so

$$
R_{0}=\frac{1}{2}\left(\frac{\sum_{i=1}^{n} \sum_{j=0}^{n} i^{2} p(i, j) \lambda_{1}}{<k_{1}>+<k_{2}>}+\frac{\sum_{i=0}^{n} \sum_{j=1}^{n} j^{2} p(i, j) \lambda_{2}}{<k_{1}>+<k_{2}>}+\sqrt{\Delta}\right)
$$

Then we can obtain the following results.

Theorem1. For the information dissemination model (2), the disease-free equilibriu $E_{0}=(\overbrace{(0,0, \cdots, 0}^{(n+1)^{2}}$ is locally asymptotically stable if $R_{0}<1$, but unstable if $R_{0}>1$.

\subsection{Global stability of the disease-free equilibrium and the existence and uniqueness of endemic equilibrium}

In order to obtain the global stability of the disease-free equilibrium $E_{0}$, we need the following lemma.

Lemma1 $\left({ }^{[10]}\right)$. Consider the system

$$
\frac{\mathrm{dy}}{d t}=\boldsymbol{A} y+H(y)
$$

Where $\boldsymbol{A}$ is an $(\mathrm{n}+1)^{2} \times(\mathrm{n}+1)^{2}$ matrix and $H(y)$ is continuously differentiable in a region $D \in R^{\mathrm{n}}$. Assume that

(1) the compact convex set $C \subset D$ is positively invariant with respect to the system (4), and $\mathbf{0} \in C$,

(2) $\lim _{y \rightarrow 0} \frac{\|H(y)\|}{\|y\|}=0$,

(3) there exists $r>0$ and a (real) eigenvector $\boldsymbol{\omega}$ of $\boldsymbol{A}^{T}$ such that $(\boldsymbol{\omega} \cdot y) \geq \mathrm{r}\|y\|$ for all $\mathrm{y} \in \mathrm{C}$,

(4) $(\boldsymbol{\omega} \cdot H(y)) \leq 0$ for all $\mathrm{y} \in C$,

(5) $\mathrm{y}=0$ is the largest positively invariant set (for(4)) contained in $G=\{\mathrm{y} \in C \mid(\omega \cdot H(y)=0)\}$. Then either $y=0$ is globally asymptotically stable in $C$, or for any $\mathrm{y}_{0} \in C-\{0\}$ the solution $\phi\left(t, y_{0}\right)$ of (4) satisfies $\liminf _{t \rightarrow \infty}\left\|\varphi\left(t, y_{0}\right)\right\| \geq m$, where $m>0$, independent of the initial value $\mathrm{y}_{0}$. Moreover, there exists a 
constant solution of (4), $\mathrm{y}=y^{*}, y^{*} \in C-\{0\}$.

Let

$$
\mathrm{y}=\left(y_{(0,0)}, \cdots, y_{(0, n)}, y_{(1,0)}, \cdots, y_{(1, n)}, \cdots, y_{(n, 0)}, \cdots, y_{(n, n)}\right)^{T} .
$$

then, equation (2) can be rewritten as a compact vector form,

$$
\frac{\mathrm{dy}}{d t}=A y+H(y)
$$

Where $A y$ is the linear part of $\mathrm{y}$, and $H(\mathrm{y}) \leq 0$ is the nonlinear part of $\mathrm{y}, H(\mathrm{y})$ is a column vector whose $\mathrm{k}_{1} \times(n+1)+\left(k_{2}+1\right) t h$ component is $-\lambda_{1} \mathrm{k}_{1} i_{\left(k_{1}, k_{2}\right)} \theta_{1}(t)-\lambda_{2} \mathrm{k}_{2}$

$i_{\left(k_{1}, k_{2}\right)} \theta_{2}(t) \cdot k_{1}, k_{2}=1, \ldots,(n+1)$.

All the hypotheses of Lemma1 are satisfied.

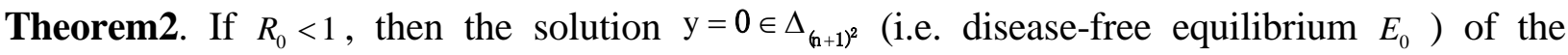
system (5) is globally asymptotically stable in $\Delta_{(n+1)^{2}}$, otherwise $R_{0}>1$, there exists a constant solution of (5), $\mathrm{y}=y^{*}, y^{*} \in \Delta_{(n+1)^{2}}-\{0\}$.

Theorem3. If $R_{0}>1$, there exists an unique endemic solution of (5), $\mathrm{y}^{*}=\left(y_{1}^{*}, y_{2}^{*}, \cdots, y_{(n+1)^{2}}^{*}\right)\left(y_{i}^{*} \neq 0, \forall i=1,2, \cdots, \quad(n+1)^{2}\right)$.

\section{Numerical simulations}

In this section, we perform numerical simulation of the system (1) by MATLAB based on BA scale-free network with $N=8000$ and $p(k, \cdot)=\gamma^{-2.1}$ : (i) The maximum of $k_{1}, k_{2}$ are both selected to 10; (ii) Let the maximum of $k_{1}, k_{2}$ are fixed to 20;

First, the total density of informed individuals with respect to the time series is simulated on BA scale-free networks.

In Figure 3, we set $\lambda_{1}=0.29$ and $\lambda_{2}=0.29$, in this case $R_{0}=0.703$, when the initial values is positive, the stable density of informed individual will be 0 ;

In Figure 4(a), we set $\lambda_{1}=0.79$ and $\lambda_{2}=0.79$, in this case $R_{0}=1.91$, when the initial values is positive, the stable density of informed individuals will be positive; In Figure 4(b), we set $\lambda_{1}=0.59$ and $\lambda_{2}=0.4$, in this case $R_{0}=1.19$, when the initial values is positive, the stable density of informed individuals will be positive; These simulations support the claim that information provided to individual will eventually die out when $R_{0}<1$, that is, information will not spread, and information provided to individual will not eventually die out when $R_{0}>1$, that is, information will spread. In figure 4(b), the total density solution $i_{\left(k_{1}, k_{2}\right)}$ of the model has a critical point, so if the initial informed individuals is less than this point, non-monotonicity of the total densities of informed individuals will display, as shown in Figure 4(b).

Figure 5 demonstrate $R_{0}$ with $\lambda_{1}, \lambda_{2}$ on BA scale-free network.

From Figure 6 we can see the influence of $\lambda_{1}$ and $\lambda_{2}$ on $R_{0}$ on BA scale-free network.

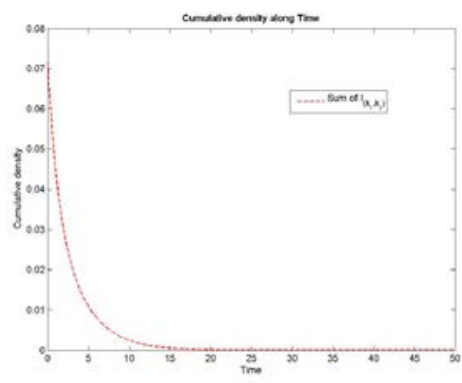

Figure3. The cumulative density of I nodes changes over the time t on BA scale-free network. $\lambda_{1}=0.29, \lambda_{2}=0.29, n=20, N=8000, R_{0}=0.703<1$. 

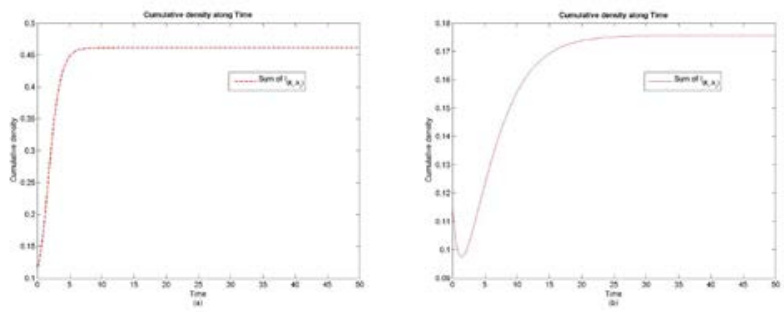

Figure4. The cumulative density of I nodes changes over the time $t$ on BA scale-free network.

(a) $n=20, \lambda_{1}=0.79, \lambda_{2}=0.79, R_{0}=1.91$; (b) $n=20, \lambda_{1}=0.59, \lambda_{2}=0.4, R_{0}=1.19$;
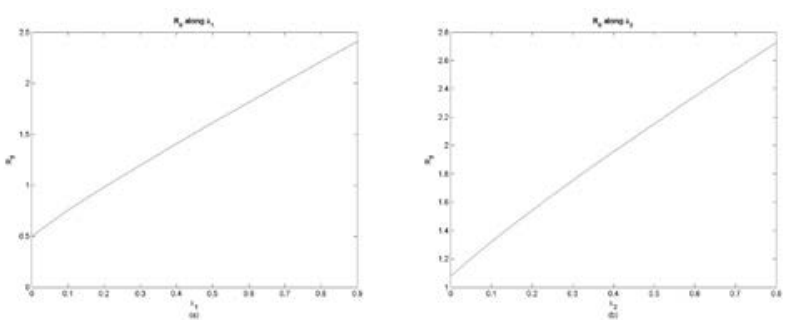

Figure5. The basic reproduction number $R_{0}$ as a function of model parameters $\lambda_{1}, \lambda_{2}$ on BA scale-free network. (a) $n=10, \lambda_{1}=0.29$; (b) $n=10, \lambda_{1}=0.56$;

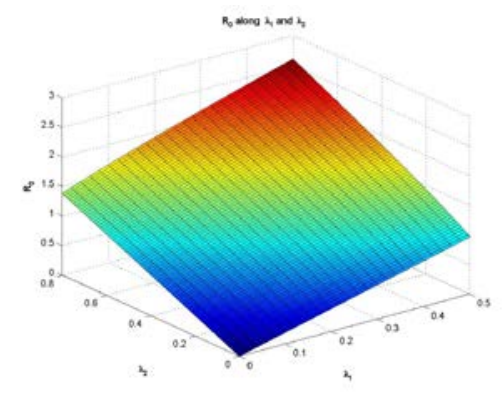

Figure6. The basic reproduction number $R_{0}$ as a function of $\lambda_{1}$ and $\lambda_{2}$ on BA scale-free network.

\section{Conclusion}

In this paper, we formulated an SIS information dissemination model based on BA sacle-free network. We have discussed the global stability of the disease-free equilibrium of the model and obtained a concrete formula of the basic reproduction number $R_{0}$, and several numerical examples have been given to verify our analytical results. These simulations support the claim that information provided to individual will eventually die out when $R_{0}<1$, that is, information will not spread, and information provided to individual will not eventually die out when $R_{0}>1$, that is, information will spread. Finally, the relationship between the basic reproduction number $R_{0}$ and model parameters $\lambda_{1}$ and $\lambda_{2}$ were illustrated. The model is suitable not only for information spreading, but also for transmission of infectious disease and computer virus etc.

\section{Acknowledgment}

The work is supported by the Youth Science Fund of Shanxi Provence (Grant No. 2011021001-2). Corresponding author. E-mail: qinpeng@nuc.edu.cn.

\section{References}

[1] Y. Moreno, M. Nekovee, and A F. Pacheco, "Dynamics of rumor spreading in complex networks,” Physical Review E, vol. 69, Jun. 2004, doi:10.1103/PhysRevE.69.066130. 
[2] A. Sudbury, "The Proportion of the Population Never Hearing a Rumour," Journal of Applied Probability, vol. 22, Jun. 1985, pp. 443-446, doi:0.2307/3213787.

[3] D H. Zanette, “Critical behavior of propagation on small-world networks," Physical Review E, vol. 64, Nov. 2001, doi:10.1103/Phys RevE.64.050901.

[4] D H. Zanette, "Dynamics of rumor propagation on small-world networks," Physical Review E, vol. 65, Mar. 2002, doi:10.1103/ Phys Rev E.65.041908.

[5] Z H. Liu, Y C. Lai, and N. Ye, "Propagation and immunization of infection on general networks with both homogeneous and heterogeneous components,” Physical Review E, vol. 67, Mar. 2003, doi: 10.1103/PhysRevE.67.031911.

[6] J. Zhou, Z H. Liu, and B W. Li, “Influence of network structure on rumor propagation,” Physics Letters A, vol. 368, Sep. 2007, pp. 458-463. doi:10.1016/j.physleta.2007.01.094.

[7] H. Kesten, and V. Sidoravicius, "The spread of a rumor or infection in a moving population," The Annals of Probability, vol. 33, Nov. 2005, pp. 2402-2462. doi:10.1214/009117905000000413.

[8] O. Diekmann, J A P. Heesterbeek, and J A J. Metz, "On the definition and the computation of the basic reproduction ratio R0 in models for infectious diseases in heterogeneous populations," Mathematical Biol, vol. 28, Jan.1990, pp. 365-382. doi:10.1007/BF00178324 .

[9] P. van den Driessche and J. Watmough, "Reproduction numbers and sub-threshold endemicequilibria for compartmental models of disease transmission,” Mathematical Biosciences, vol. 180, Nov. 2002, pp. 29-48, doi:10.1016/S0025-5564(02)00108-6.

[10] A. Lajmanovich, and J.A. Yorke, "A deterministic model for gonorrhea in a nonhomogeneous population,” Mathematical Biosciences. vol. 28, 1976, pp. 221-236, doi:10.1016/0025-5564(76) 90125-5. 